\title{
Variability of Clinical and Paraclinical Manifestations in Antiphospholipid Antibody Syndrome - Two Case Reports and Literature Review
}

\author{
Elena Laura Iliescu ${ }^{1 *}$, Letitia Toma ${ }^{1}$, Simona Ioanitescu ${ }^{1}$, Vlad Herlea ${ }^{2}$, Mihai Dodot ${ }^{1}$, Teodora Isac $^{1}$, Mihaela \\ Grumeza $^{1}$ and Adriana Mercan-Stanciu ${ }^{1}$ \\ ${ }^{1}$ Department of Internal Medicine II, Fundeni Clinical Institute, Romania
}

${ }^{2}$ Department of Pathology, Fundeni Clinical Institute, Romania

Submission: October 10, 2018; Published: October 29, 2018

*Corresponding author: Dr. Elena Laura Iliescu, Department of Internal Medicine II, Fundeni Clinical Institute, Bucharest 022328, Romania.

\begin{abstract}
Antiphospholipid antibody syndrome is a well- known, although rare, autoimmune condition characterized by thromboembolic events and/ or pregnancy morbidity, in the presence of antiphospholipid antibodies. We present the cases of two women (aged 59 and 37 years old), both diagnosed with this disease, but having different forms of manifestation; the first one consists of recurrent episodes of pulmonary embolies and renal impairment, with relatively late onset, while the second case describes early history of pregnancy morbidity in a young patient that also presents kidney function alterations; both patients have suggestive serology. A kidney biopsy was performed in both cases. The particularity of antiphospholipid syndrome as a pathological entity consists in its clinical variability and heterogenicity, therefore, whenever there are signs of thrombosis or pregnancy complications, this condition must be suspected and further investigated.

Keywords: Antiphospholipid Antibody Syndrome; Lupus Anticoagulant; Anticardiolipin Antibodies;Pulmonary Embolism; Pregnancy Morbidity

Abbreviations: APS: Antiphospholipid Antibody Syndrome; aPL: Antiphospholipid Antibodies; LA: Lupus Anticoagulant; aCL: Anticardiolipin; aß2GPI: anti- $\beta 2$ Glycoprotein-I Antibodies; DVT: deep vein thrombosis(DVT); PE: Pulmonary Embolism (PE); AST: Aspartate Transaminase; ALT: Alanine Transaminase; CK:Creatine Kinase; LDH: Lactate Dehydrogenase; ESR: Erythrocyte Sedimentation Rate; APTT: Activated Partial Thromboplastin Time; INR: International Normalized Ratio; ANCA: Anti-Neutrophil Cytoplasmic Antibodies;SSA: Anti-Sjogren's Syndrome A; SSB: Anti-Sjogren's Syndrome B; ANA: Antinuclear Antibodies; dsDNA: Anti-double Stranded DNA Antibodies; RF: Rheumatoid Factor; UFH: Unfractionated Heparin; SLE: Systemic Lupus Erythematosus
\end{abstract}

\section{Introduction}

The antiphospholipid antibody syndrome (APS) or Hughes's syndrome is a well-known autoimmune condition, that consists in the association of antiphospholipid antibodies (aPL) and a status of hypercoagulability, thus leading to the occurrence of thromboembolic complications and/ or pregnancy morbidity [1]. It was first described in 1983 by Graham RV Hughes, as a complex pattern of features, including thrombotic events, recurrent spontaneous abortions, neurological disease and the presence of lupus anticoagulant [2,3].

The clinical manifestations of APS are heterogeneous and nonspecific, therefore the detection of circulating antiphospholipid antibodies is essential for the diagnosis. The most common subgroups of antibodies involved in the APS include lupus anticoagulant (LA), anticardiolipin (aCL) and anti- $\beta 2$ glycoprotein-I antibodies (aß2GPI) (either IgG or IgM) [4]. In order to establish a proper diagnosis of the antiphospholipid antibody syndrome, the international consensus states that presence of at least one clinical manifestation (vascular thrombosis or pregnancy complication) and one biological finding is required. Furthermore, the laboratory abnormality must be observed repeatedly (on two or more occasions), with a minimum of 12 weeks in between the positive determinations $[5,6]$.

Vascular thrombosis may be diagnosed by imaging techniques, Doppler studies or histopathological findings and it most commonly includes lower limb deep vein thrombosis (DVT) and/ or pulmonary embolism (PE), although any part of the venous system may be affected by the pathogenic process, including the renal, portal, mesenteric, cerebral or superficial veins. It can also include arterial thrombosis, affecting especially the intracranial vasculature, thus resulting in transient cerebral ischaemia or even stroke [1]. Myocardial infarction may appear as well, although it is considered to be a less common manifestation [7]. 


\section{Orthopedics and Rheumatology Open Access Journal (OROAJ)}

\section{Case Reports}

\section{Results}

The first case we describe is that of a 59-year-old woman, with a history of arterial hypertension (developed after the third child birth), gout, hypothyroidism (under substitutive hormonal therapy); she used to be a heavy smoker (40 pack-years), but she had admittedly quit for about 10 years. She had no personal history of premature birth or abortion. This patient presented to the hospital accusing fatigability, weakness, shortness of breath and discrete chest pain - symptoms that were persistent for about two weeks; she also complained about pain and stiffness affecting the hand joints bilaterally, as well as both knees, associated with low lumbar pain and important functional impairment. The clinical examination revealed an obese patient, with normal coloration of the teguments and mucosae and no signs of cyanosis or oedema; she had stiffness and swelling of the hand and finger joints bilaterally, with discrete pain at this level. It is important to mention that the patient was unable to perform daily tasks by herself, such as dressing, and undressing and she presented functional difficulties when asked to position in dorsal decubitus.

Auscultation of the heart and lungs did not reveal any modifications. Her blood pressure was $150 / 100 \mathrm{mmHg}$ and her heart rate was 95 beats per minute. The laboratory tests revealed a mildly elevated aspartate transaminase (AST) of $62 \mathrm{U} / \mathrm{l}$, but with normal alanine transaminase (ALT). Important elevation of creatine kinase $(\mathrm{CK})$ was noted, of about three times the normal value $(1125 \mathrm{U} / \mathrm{L})$, associated with a slight increase in CK-MB (36 U/L) and in lactate dehydrogenase (LDH) blood levels Serum creatinine was increased $(1.81 \mathrm{mg} / \mathrm{dl})$, as well as serum urea $(89.1 \mathrm{mg} / \mathrm{dl})$. There was an important elevation in the blood levels for fibrinogen $(813 \mathrm{mg} / \mathrm{dl}), \mathrm{C}$ reactive protein $(117$ $\mathrm{mg} / \mathrm{l})$ and erythrocyte sedimentation rate (ESR) $(68 \mathrm{~mm} / \mathrm{h})$. Laboratory tests also revealed mild leucocytosis (9.53 x103/ ul) predominantly with neutrophils, a haemoglobin level of 12.6 $\mathrm{g} / \mathrm{dl}$ and an increased number of platelets (488 x103/ ul). An electrocardiogram was soon performed, describing sinus rhythm with sinus tachycardia, with QRS axis at -10 degrees and negative $\mathrm{T}$ waves in V1, V2 and V3 (but no acute changes were noted, by comparison with the previous examinations of the patient). The chest X-ray revealed accentuated peribronchovascular interstitium, enlargement of the pulmonary hilum on both sides and cardiomegaly. The patient also underwent a transthoracic echocardiography that indicated discrete dilation of the right heart cavities and moderate tricuspid valve regurgitation, being otherwise normal. The alterations in creatinine and urea blood levels, associated with the positivity for proteins in the urine dipstick test (performed at admission), required a more detailed evaluation of the renal function, therefore the blood tests were followed by a quantitative determination of the 24-hour proteinuria, revealing a value of $0.7 \mathrm{~g}$ of protein per day.
Because a suspicion of pulmonary embolism was raised, D-dimers were measured and found to be increased (1400 ng/ $\mathrm{ml}$ ). The activated partial thromboplastin time (APTT) was normal, with normal prothrombin time and normal International Normalized Ratio (INR). For further evaluation, a circulating anticoagulant antibodies panel was also performed, showing the presence of lupus anticoagulant. Anticardiolipin and anti- $\beta 2$ glycoprotein- I antibodies were not detected in the patient's serum. Serology for anti-neutrophil cytoplasmic antibodies (ANCA) was negative. In addition, laboratory tests revealed negativity for: Anti- Sjogren's Syndrome A (SSA), Anti-Sjogren's Syndrome B (SSB), antinuclear antibodies (ANA), anti-double stranded DNA antibodies (dsDNA), Rheumatoid Factor (RF), and anti-glomerular basement membrane antibody.

Computerized tomography of the thorax with PE protocol was urgently performed, describing partial filing defects within the segmental pulmonary arteries of the inferior right lobe, as well as sequellary micro-nodules located in both lungs and axillary and mediastinal lymphadenopathies; a small right pleural effusion was also detected. Venous Doppler ultrasound examination of the legs showed no signs of thrombosis on this level.

The patient was administered parenteral anticoagulation with enoxaparin for 14 days, followed by oral treatment with rivaroxaban (20 mg daily), for the PE. Furthermore, given the renal impairment, the positivity for lupus anticoagulant, as well as the pain and swelling of several joints (that did not improve

with the use of nonsteroidal anti-inflammatory drugs), the patient was given corticosteroid pulse therapy with one gram of methylprednisolone per day, for three days, followed by oral therapy with $4 \mathrm{mg}$ of dexamethasone daily. Under therapy, the clinical status of the patient improved significantly, while creatinine and urea slowly normalized, as well as CK, CK-MB, ALT, AST, fibrinogen and C reactive protein. The ESR decreased to 15 $\mathrm{mm} / \mathrm{h}$, platelets count was normal, while leucocytosis persisted, within two weeks of treatment. The patient was discharged, with the recommendation of continuing both anticoagulant therapy with rivaroxaban and corticosteroid therapy with dexamethasone, until the next medical assessment.

Therefore, the patient presented again to the hospital after a month of treatment; at the moment of admission, she was in good clinical condition and only accused mild fatigability and asthenia. Clinical examination was normal and the ECG was comparable to the previous one. However, D-dimers had a value of $1600 \mathrm{ng} / \mathrm{ml}$ and there was also an increase of serum creatinine level $(1.52$ $\mathrm{mg} / \mathrm{dl})$ and urea $(87 \mathrm{mg} / \mathrm{dl})$. Blood tests revealed leucocytosis of $16.45 \times 103 /$ ul, predominantly with neutrophils, a haemoglobin level of $12.7 \mathrm{~g} / \mathrm{dl}$ and a normal number of platelets (289 x103/ ul). The 24-hour proteinuria was also determined and was importantly elevated ( $3.28 \mathrm{~g}$ of proteins per day), by comparison with the previous determination. 
The patient underwent a second computerized tomography of the thorax, for control purposes, which revealed the remission of the right segmental pulmonary embolism, while a new filling defect located within the segmental pulmonary arteries of the superior left lobe was described. The oral anticoagulation, that she was admittedly taking daily at home with no exception, was immediately replaced by parenteral anticoagulation with unfractionated heparin (UFH). Also, while at hospital, she underwent a second episode of pulse therapy with methylprednisolone per day, for three days; oral dexamethasone was not reintroduced in the therapeutic scheme after the pulse therapy; instead, it was replaced with $16 \mathrm{mg}$ of methylprednisolone orally daily.

In order to determine the cause of the renal impairment in the presence of lupus anticoagulant, a kidney biopsy was performed, without complications. Optical microscopy exploration described a fragment consisting in 7 glomeruli, with ischemic aspect, without mesangial proliferation, as well as rare tubules with pseudo-thyroidian appearance, interstitial sclerosis and atrophic tubules, hyaline nodular arteriolar deposits, narrowing in the arteriolar lumen, arteriolar media hypertrophy; there were also spotted arterial vessels with lamellar structure of the internal elastic lamina and discrete diffuse interstitial inflammation. (Figures 2-4). Electronic microscopy evaluation showed glomerulus with permeable capillaries, with some glomerular loops presenting ischemic basal membranes and important narrowing, in association with discrete signs of endotheliosis.

She was discharged in good condition and was recommended enoxaparin 4000 UI twice a day. One month after the second $\mathrm{PE}$, she presented again to the hospital, for re-evaluation, with goodclinical status; the control CT scan of the thorax showed no signs of pulmonary embolism. D-dimers, as well as all the other laboratory tests (hemogram, coagulation tests) were repeated and were within normal range, but the serum abnormalities of creatinine and proteinuria persisted, although there was a significant improvement: creatinine was $1.39 \mathrm{mg} / \mathrm{dl}$, while the 24 - hour proteinuria had a value of $0.3 \mathrm{~g}$. She was discharged from hospital after another corticosteroid pulse therapy, with the recommendation of enoxaparin 4000 UI twice a day (in order to prevent other thromboembolic events), and $16 \mathrm{mg} /$ day of methylprednisolone.

On three monthsfollow up, the presence oflupus anticoagulant was verified again, and the initial diagnosis of antiphospholipid antibodies syndrome was confirmed. She was therefore advised to indefinitely continue the anticoagulant treatment and the corticoid therapy.The second case describes a 37-year-old female, that presented to the hospital for fatigability, weakness, nausea, dizziness, cervical pain, nose bleeding and paresthesia of the right side of the body. The symptoms were persistent within the last month and became progressively aggravated two days prior to the admission. The anamnesis of the patient revealed the following information: at 19 years old, she willingly underwent three abortion procedures (the third one revealing intra-uterine fetal death); the forth pregnancy (at 21 years old) was categorized as having a high risk of abortus imminens (at 6 months of pregnancy), but she managed to give birth, on term, to a perfectly healthy baby; after the first childbirth, the woman was diagnosed with somatoform disorder, for which she received specific psychiatric therapy; she soon had a fifth pregnancy (at 23 years old) during which she presented with vaginal bleeding, associated with pregnancy- induced hypertension, generalized oedema (in the eighth month); she received tocolytic treatment and she gave birth naturally, on term, to a dead baby, after experiencing another intra-uterine fetal death; this episode was followed by a sixth pregnancy (approximately nine months after thefifth), during which the patient presented to the hospital with perioral and palmar cyanosis and haemorrhage within the second month; she received progesterone based treatment, but within the fourth month of pregnancy, she accused shortness of breath, chest pain and hypertension; ultrasound examination revealed another intra-uterine fetal death.

Clinical examination of the patient, at the moment of admission to our hospital, was normal. Blood test showed normal leucocytes and platelets count, a normal haemoglobin of $12 \mathrm{~g} / \mathrm{dl}$. Liver function was normal. She had $1.7 \mathrm{~g}$ proteinuria per 24 hours and serum creatinine was $1.59 \mathrm{mg} / \mathrm{dl}$. Serum complement levels were low. The APTT was abnormal (50 seconds, while the laboratory limits were set between 26 and 40 seconds), with a normal prothrombin time and normal INR.In view of the personal history of pregnancy morbidity, a complete antinuclear antibody profile was done, showing strong positivity for the lupus anticoagulant, as well as for anticardiolipin antibody, while aß2GPI antibodies and dsDNA antibody were negative. Electrocardiogram, upper gastrointestinal endoscopy and ultrasound examination of the abdominal and pelvic region were normal.

The patient underwent a kidney biopsy consisting in two fragments. The first one was represented by adipose tissue, with focal collagenous fibrosis. The second one described 13 glomeruli, with important alterations in the histologic architecture, due to the presence of multiple micro-thromboses that induced variable degrees of glomerular atrophy; there were also atrophy regions within the tubules structure, as well as interstitial fibrosis located predominantly in proximity to the glomeruli, with positivity in Masson coloration (Figures 1-4).The diagnosis suspicion was of primary antiphospholipid antibody syndrome with renal involvement and she was put on oral treatment with $75 \mathrm{mg}$ of Aspirin per day, being discharged in good clinical condition after 5 days of hospitalization. On three months follow up, the lupus anticoagulant and anticardiolipin antibody were positive. 


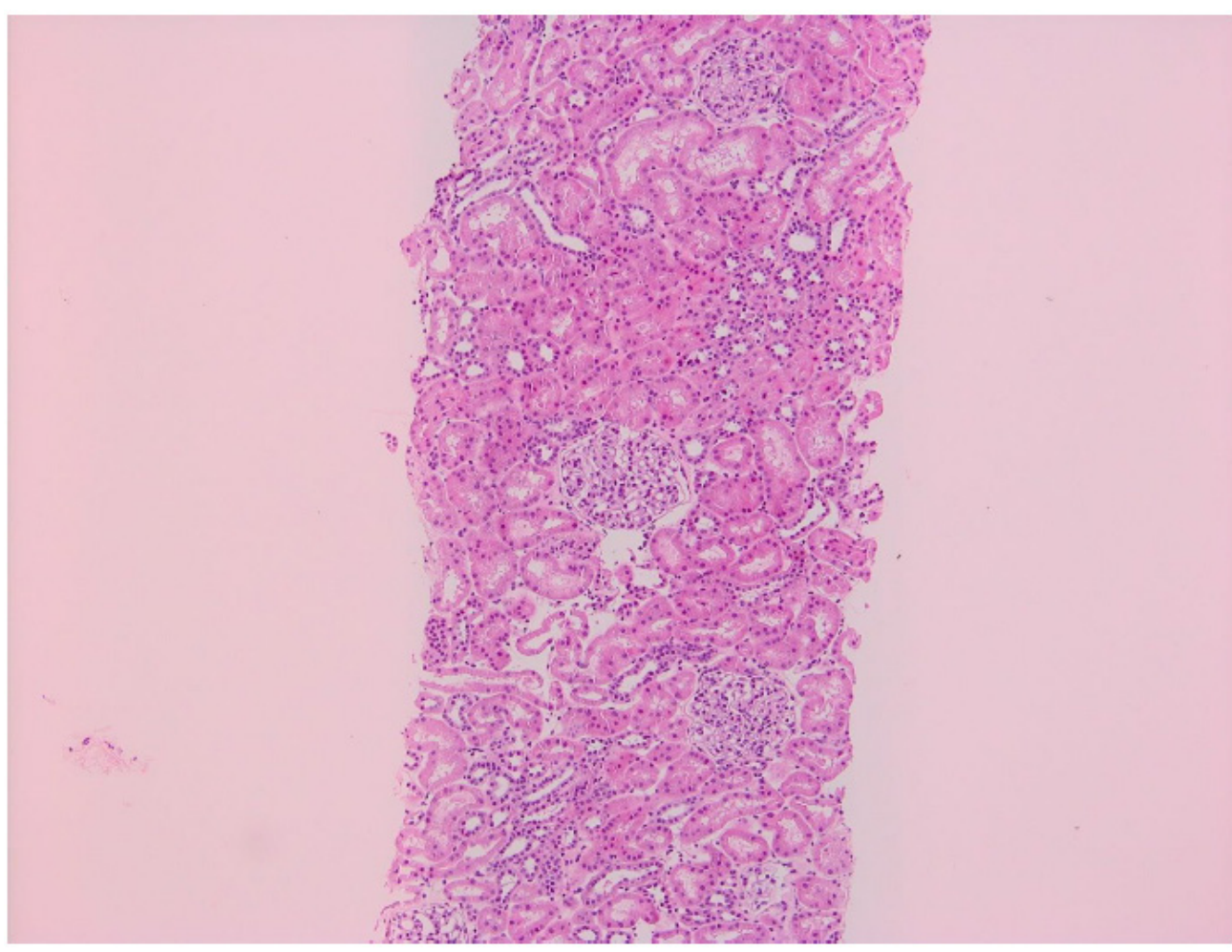

Figure 1: Microscopic view of the kidney biopsy specimen, using conventional hematoxylin-eosin (HE), atx100 magnification, revealing corpuscles with obliterated Bowmann's space and alterations of architecture.

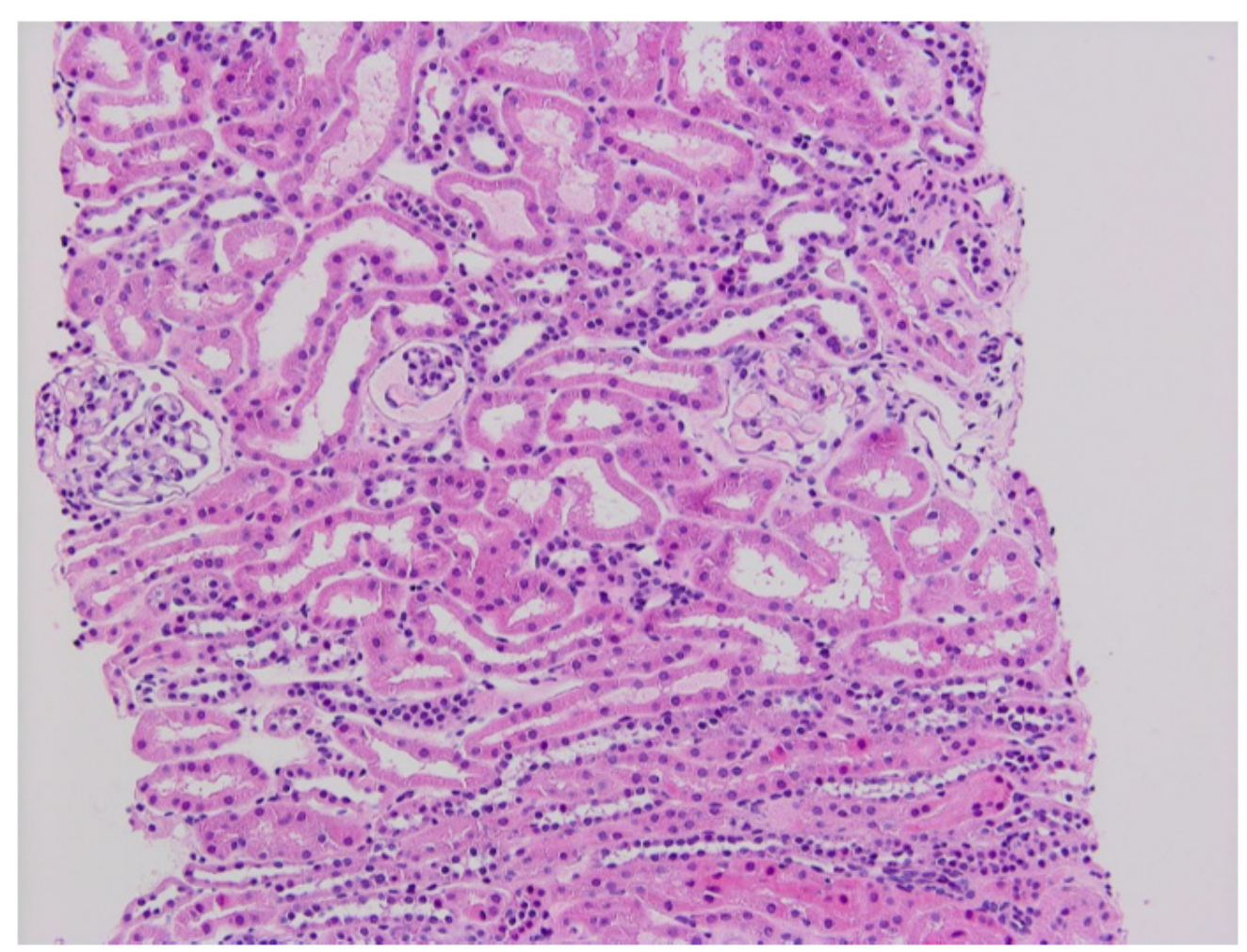

Figure 2: Microscopic view of the kidney biopsy specimen, using conventional hematoxylin-eosin (HE), atx200 magnification, revealing altered glomerular architecture. 


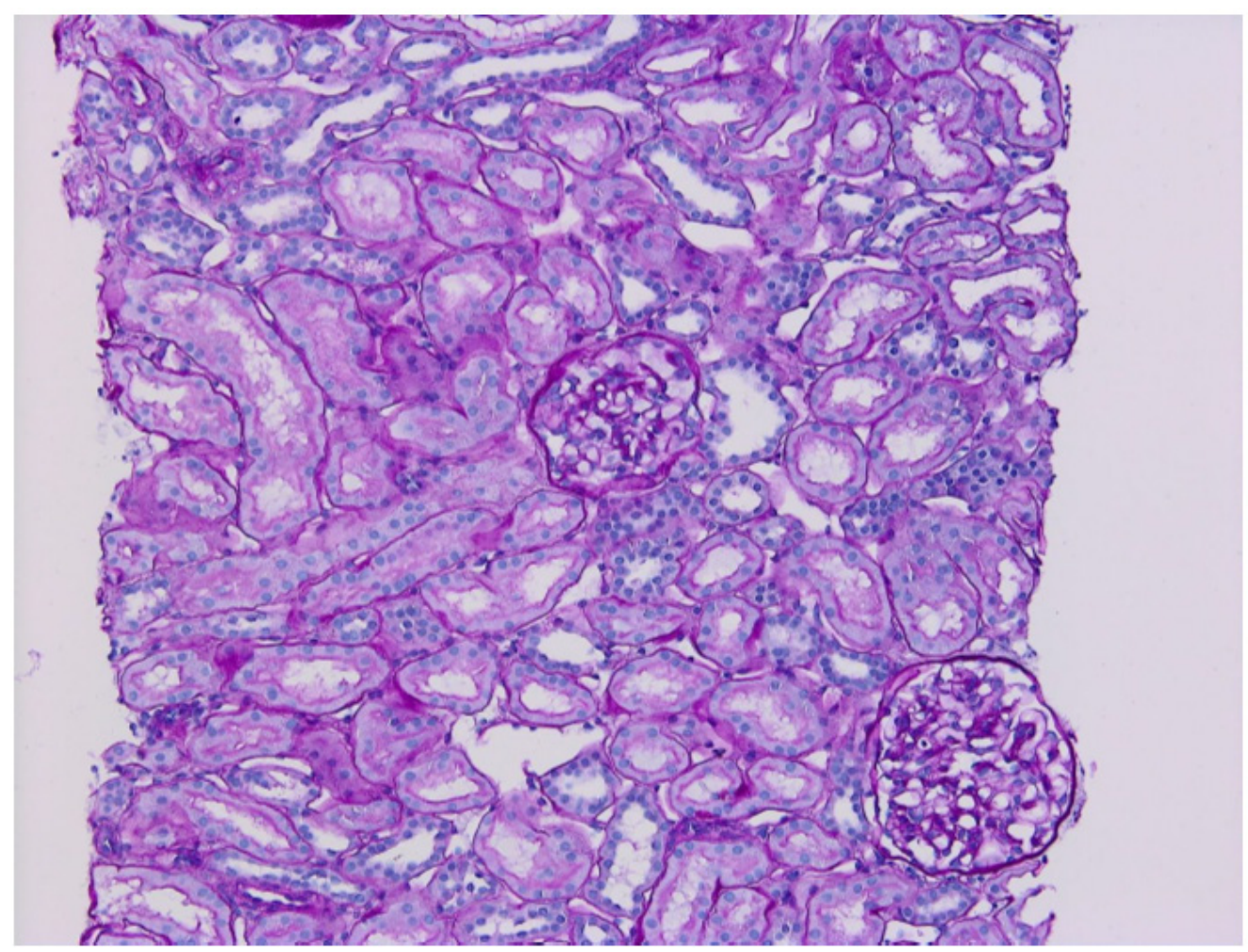

Figure 3: Microscopic view of the kidney biopsy specimen, using Periodic acid-Schiff (PAS) stain, at x200magnification showing obliterated Bowmann's space and glomerular sclerosis.

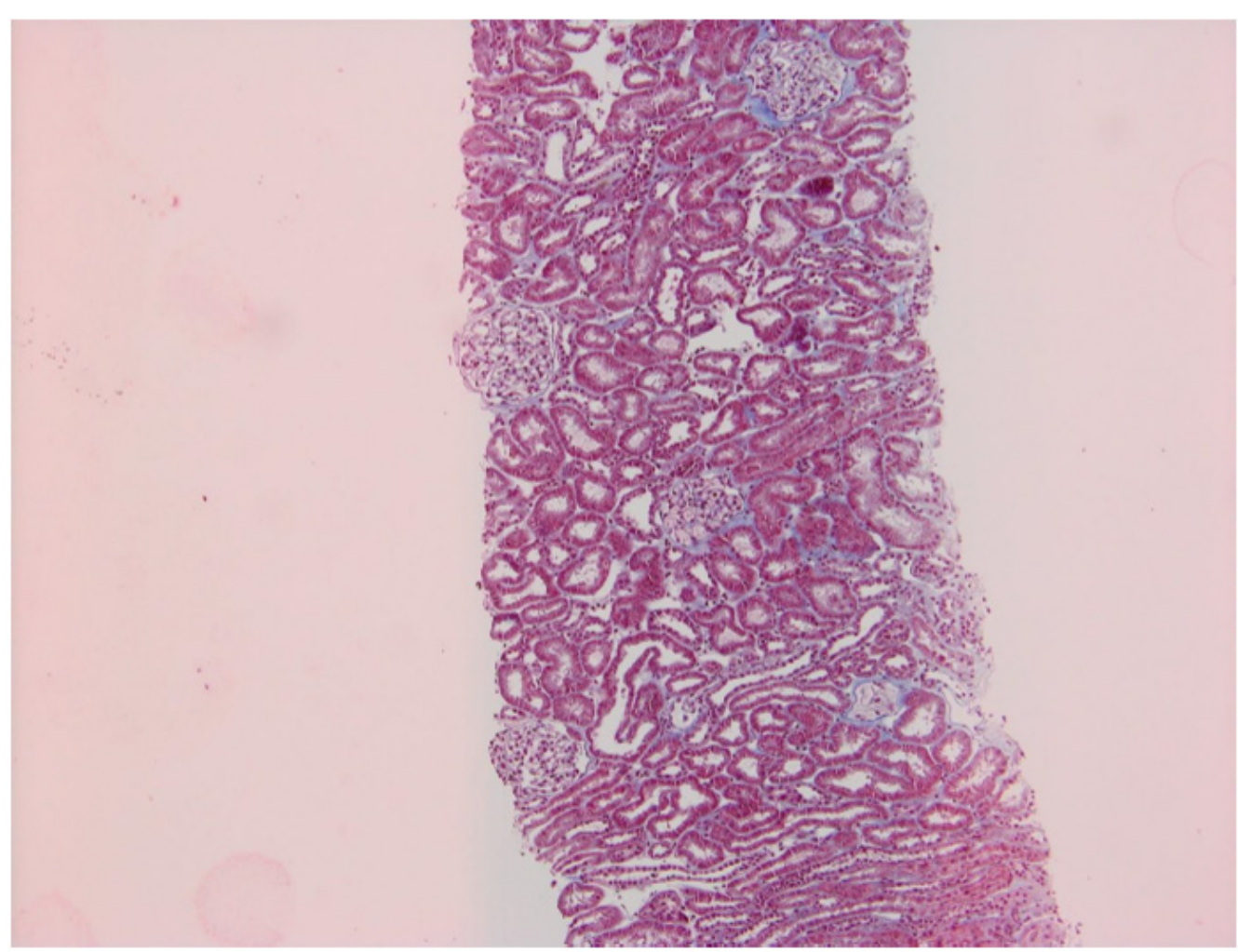

Figure 4: Microscopic view of the kidney biopsy specimen, using Masson coloration, at x100 magnificationshowing moderate interstitial fibrosis with periglomerular disposition. 


\section{Orthopedics and Rheumatology Open Access Journal (OROAJ)}

\section{Discussion}

In this paper, we report two cases of antiphospholipid antibodies syndrome, that occur and manifest differently in two women aged 59, and respectively 37 , an aspect that is persistent with the literature data, showing how clinical presentation of this pathology has important variations. Within the last 20 years, there have been many epidemiological studies concerning APS, that have demonstrated its occurrence in many different populations and ethno-geographical groups $[8,9]$. The frequency and clinical complications of APS in individuals may differ, due to environmental or genetic factors, or both [10].

Although APS was first described in the presence of systemic lupus erythematosus (SLE), more than half of the patients diagnosed with APS do not present clinical, nor biological evidence of another autoimmune condition, being therefore classified as having primary antiphospholipid syndrome [11]. This is the case of both the aforementioned patients, none of which had another objective finding of autoimmune disease, excepting the antiphospholipid syndrome, that presented differently. In the first case, the patient had recurrent pulmonary embolism, while the second patient described multiple episodes of pregnancy associated morbidity; however, in both cases, important renal involvement was reported, that manifested with proteinuria and nitrogen retention. While many visceral implications of primary APS have been characterized since its first description in 1983, the renal manifestations of this pathological entity were underestimated and have received scarce attention until recently $[12,13]$. Not only can the kidney be affected by the APS, but it also represents a major scene in the pathogenesis of this condition. Moreover, renal involvement is a frequent characteristic of the catastrophic APS. Also, there is evidence of the presence of antiphospholipid antibodies in patients with end-stage renal failure [14].

The pathway by which antiphospholipid antibodies determine thrombotic events remains yet unclear, although it may involve interactions with beta 2 -glycoprotein 1 , resulting in endothelial cells activation, in association with antithrombin III inhibition, protein $\mathrm{C}$ activation, inhibitory effects on fibrinolysis and interference with tissue factor and thrombin [15]. Vascular injuries, even minimal, as well as transient hypotension may also be involved in microthrombi formation [16].

Therapeutic approach of APS must be focused on three main aims: first of all, identification and optimal treatment of precipitating factors must be realized; prevention and treatment of thrombotic events is vital and also, the excessive activity of cytokines must be suppressed. Therefore, optimal therapy may include: anticoagulants, corticosteroids, plasma exchange, administration of intravenous gamma-globulins and, if necessary, cyclophosphamide [17].The first patient we presented had a very particular evolution, under treatment. She received anticoagulation therapy after the diagnosis of the first $\mathrm{PE}$, consisting in parenteral anticoagulation withenoxaparin for
14 days, followed by oral treatment with rivaroxaban $(20 \mathrm{mg}$ daily) at discharge. While on rivaroxaban, which is known to be an inhibitor of the platelet activation, as well as of the fibrin clot formation (by inhibiting factor Xa in both intrinsic and extrinsic coagulation pathways), she developed a second PE, with different location from the first one. There are several potential causes reported in the literature for the recurrence of thrombotic events, despite using anticoagulant therapy, such as: malignant tumours [18], myeloproliferative disorders (polycythemiavera and essential thrombocythemia) [19], vasculitis (Behcet disease), paroxysmal nocturnal haemoglobinuria, pregnancy, vascular abnormalities (for example the venous compression in MayThurner syndrome) and last, but not least, the antiphospholipid syndrome itself may be a cause of anticoagulant therapy failure $[20,21]$.

The mechanism by which APS induced the occurrence of a second pulmonary embolism in our first patient may involve disruption in annexin $V$ shield structure, neutrophil extracellular traps, activation of the endothelial cells, protein $\mathrm{C}$ inhibition or fibrinolysis [21]. However, there is evidence of recurrent thrombotic events while on rivaroxaban even in individuals that do not present with any of the aforementioned conditions, especially in obese patients, although the cause remains unclear [22]. For treating the second thrombo-embolic event, she was given parenteral anticoagulation with unfractionated heparin, followed by enoxaparin (that the patient had to learn how give herself, in order to continue the treatment after discharge). This second therapeutic approach turned out to be more efficient, as the patient did not repeat any thrombotic events, while on enoxaparin. In terms of reducing cytokines' activity, corticosteroid treatment was applied, in monthly pulse-therapy, followed by daily oral administration. The first pulse therapy was followed by $4 \mathrm{mg}$ of dexamethasone daily, under which there was initially a significant improvement of the paraclinical parameters. However, since the renal impairment persisted, dexamethasone was replaced by oral treatment with $16 \mathrm{mg}$ of methylprednisolone daily. Kidney biopsy was considered to be necessary in this patient's case, given the renal impairment in the presence of lupus anticoagulant, showing important alterations in the glomeruli structure, as well as modifications of the vessels, although no clear elements of vasculitis in the analysed fragments were spotted (most probably, due to the immunosuppressive treatment the patient had been taking a month prior to this investigation).

The second case describes a different clinical presentation of APS, characterized by pregnancy morbidity and the positivity of lupus anticoagulant and anticardiolipin antibody, on two different occasions (separated by a 3 months' time). Pregnancy morbidity may be caused by thrombosis affecting the placental bed, but the early losses may be explained by other mechanisms [1]. The antiphospholipid antibodies affect the trophoblasts directly [23]. Literature data also states that complement is 
activated in pregnancy failure [24,25]. This partially explains the efficacy of heparin therapy in the prevention of early foetus losses [1, 24-26]. As the patient showed no signs of thrombosis, anticoagulation was unnecessary, and the patient was recommended anti-platelet aggregation therapy.

\section{Conclusion}

The antiphospholipid antibody syndrome is a condition that should be suspected and properly investigated whenever there are signs and symptoms of thrombotic events and/ or pregnancy morbidity. The particularity of APS consists in its clinical variability, as different patients can have different manifestations, resulting in a diversity of clinical presentations. Renal involvement may be present in many and should be carefully investigated. A renal biopsy should be performed for further characterization, when considered necessary and the therapeutic management should involve a multidisciplinary team.

\section{Conflict of Interest}

The authors declare that they do not have anything to disclose with respect to this manuscript.

\section{References}

1. Keeling D, Mackie I, Moore GW, Greer IA, Greaves M (2012) British Committee for Standards in Haematology. Guidelines on the investigation and management of antiphospholipid syndrome. $\mathrm{Br} \mathrm{J}$ Haematol 157(1):47-58.

2. Hughes GR(1983) Thrombosis, abortion, cerebral disease and lupus coagulant. BrMedJ 187: 287(6399): 1088-1089.

3. Kawczyk-Krupka A, Cieslar G, Dubik K, Dubik A, Sieron A (2013) Primary antiphospholipid syndrome - case report. AnnAcad Med Siles 67(1):78-83.

4. Variability of clinical and paraclinical manifestations in antiphospholipid antibody syndrome - Two case reports and literature review.

5. An GD, Lim HH, Han JY (2017) Laboratory Diagnosis of Antiphospholipid Syndrome. Clin Exp ThrombHemost 3(1):2-7.

6. Miyakis S, Lockshin MD, Atsumi T, Branch DW, Brey RL, et al. (2006) International consensus statement on an update of the classification criteria for definite antiphospholipid syndrome (APS). J ThrombHaemost 4(2):295-306.

7. Bertolaccini ML, Amengual O, Andreoli L, Atsumi T, Chighizola CB, et al. (2014) $14^{\text {th }}$ International Congress on Antiphospholipid Antibodies Task Force. Report on antiphospholipid syndrome laboratory diagnostics and trends. Autoimmun Rev 13(9):917-930.

8. Sacre K, Brihaye B, Hyafil F, Serfaty JM, Escoubet B, et al. (2010) Asymptomatic myocardial ischemic disease in antiphospholipid syndrome: a controlled cardiac magnetic resonance imaging study. Arthritis and Rheumatism 62(7): 2093-2100.
9. Wilson WA (2000) Ethnicity and APS. J Autoimmun 15(2):153-155.

10. Font J, Lopez-Soto A, Cervera R, Balasch J, Pallares L, et al. (1991) The'primary' antiphospholipid syndrome: antiphospholipid antibody pattern and clinical features of a series of 23 patients. Autoimmunity9(1):69-75.

11. Horita T, Merrill JT (2004) Genetics of antiphospholipid syndrome. CurrRheumatol Rep6:458- 462.

12. Cervera R, Piette JC, Font J, Shoenfeld Y, Camps MT, et al. (2002) EuroPhospholipid Project Group: Antiphospholipid syndrome: Clinical and immunologic manifestations and pattern of disease expression in a cohort of 1,000 patients. Arthritis Rheum 46(4): 1019-1027.

13. Uthman I, Khamashta M (2006) Antiphospholipid syndrome and the kidneys. Semin Arthritis Rheu1: 360-367.

14. Nzerue CM, Hewan-Lowe K, Pierangeli S, Harris N (2002) Black swan in the kidney: Renalinvolvement in the antiphospholipid syndrome. Kidney Int 62(3): 733-744.

15. Piette JC, Cacoub P, Wechsler B (1994) Renal manifestation of the antiphospholipid syndrome. Semin Arthritis Rheum 23(6): 357-366.

16. Levine JS, Branch DW, Rauch J (2002) The antiphospholipid syndrome. N Engl J Med346(10):752- 763.

17. Bordin G, Boldorini R, Meroni PL (2003) The two hit hypothesis in the antiphospholipid syndrome: acute ischaemic heart involvement after valvular replacement despite anticoagulation in a patient with secondary APS. Lupus12(11):851-853.

18. Asherson RA, Cervera R, de Groot PG, Erkan D, Boffa MC, et al. (2003) Catastrophic antiphospholipid syndrome: international consensus statement on classification criteria and treatment guidelines. Lupus. 12(7): 530-534.

19. Falanga A, Marchetti M, Russo L (2015) The mechanisms of cancerassociated thrombosis. Thromb Res. 135(Suppl 1):S8-S11.

20. Passamonti F, Caramazza D, Mora B, Casalone R, Maffioli M (2014) It is time to change thrombosis risk assessment for PV and ET? Best Pract Res Clin Haematol 27(2):121-127.

21. Willis R, Pierangeli SS (2011) Pathophysiology of the antiphospholipid antibody syndrome. Auto Immun Highlights2(2):35-52.

22. Schulman S (2017) How I treat recurrent venous thromboembolism in patients receiving anticoagulant therapy. Blood 129(25): 3285-3293.

23. Ahmed TS, Tewari P, Kaur J, Rizvi S, Nafsi T (2016) Recurrence of Pulmonary Embolism While on Rivaroxaban: Treatment Failure. Chest Journal 149(4).

24. Nelson SM, Greer IA (2008) The potential role of heparin in assisted conception. Human Reproduction Update 14(6): 623-645.

25.Salmon JE, Girardi G (2004) The role of complement in the antiphospholipid syndrome. Current Directions in Autoimmunity. 7: 133-148.

26. Oku K, Atsumi T, Bohgaki M, Amengual O, Kataoka H, et al. (2009) Complement activation in patients with primary antiphospholipid syndrome. Ann Rheum Dis68(6): 1030-1035. 
CC This work is licensed under Creative CC (U) Commons Attribution 4.0 License BY DOI: 10.19080/OROAJ.2018.13.555855
Your next submission with Juniper Publishers will reach you the below assets

- Quality Editorial service

- Swift Peer Review

- Reprints availability

- E-prints Service

- Manuscript Podcast for convenient understanding

- Global attainment for your research

- Manuscript accessibility in different formats

( Pdf, E-pub, Full Text, Audio)

- Unceasing customer service

Track the below URL for one-step submission https://juniperpublishers.com/online-submission.php 